BULLETIN Bulletin hispanique

HISPANIQUE Université Michel de Montaigne Bordeaux

116-1 | 2014

Varia

Censures et manipulations dans les mondes ibérique et hispano-américain

Presses Universitaires François Rabelais, 2013

Geneviève Champeau

(2) OpenEdition

Journals

Édition électronique

URL : http://journals.openedition.org/bulletinhispanique/3333

DOI : 10.4000/bulletinhispanique.3333

ISSN : $1775-3821$

Éditeur

Presses universitaires de Bordeaux

Édition imprimée

Date de publication : 1 juin 2014

Pagination : 464-467

ISBN : 978-2-86781-931-5

ISSN : 0007-4640

Référence électronique

Geneviève Champeau "Censures et manipulations dans les mondes ibérique et hispano-américain »,

Bulletin hispanique [En ligne], 116-1 | 2014, mis en ligne le 01 juin 2014, consulté le 23 septembre 2020.

URL : http://journals.openedition.org/bulletinhispanique/3333 ; DOI : https://doi.org/10.4000/

bulletinhispanique.3333

Ce document a été généré automatiquement le 23 septembre 2020

Tous droits réservés 


\title{
Censures et manipulations dans les mondes ibérique et hispano-américain
}

Presses Universitaires François Rabelais, 2013

\author{
Geneviève Champeau
}

\section{RÉFÉRENCE}

Censures et manipulations dans les mondes ibérique et hispano-américain, Édition de JeanLouis Guereña y Mónica Zapata, Presses Universitaires François Rabelais, 2013, 388 p.

1 Ce deuxième volet d'une recherche collective engagée par le CIREMIA (intégré au laboratoire Interactions Culturelles et Discursives, Université François Rabelais de Tours) fait suite à Figures de la censure dans les mondes hispanique et ibéro-américain publié en 2009 chez Indigo. Il réunit 17 articles de chercheurs français, espagnols et anglais, dont 12 sont rédigés en français et cinq en espagnol. Une bibliographie et un index onomastique complètent ce volume dédié à Marie-Aline Barrachina qui consacra ses recherches à l'histoire culturelle de l'Espagne contemporaine.

2 La censure est un champ d'investigation abondamment cultivé comme l'atteste la bibliographie finale de même que, par ailleurs, la revue en ligne Represura consacrée à la guerre civile espagnole et à la période franquiste. Le volume en question ne se limite, néanmoins, ni à l'Espagne, ni à la culture en contexte dictatorial, bien que six articles concernent directement cette question, que ce soit en Espagne, dans le Portugal de Salazar, en Uruguay ou en Argentine. La notion de censure est élargie à toutes formes d'entrave à la liberté d'expression, qu'elle soit le fait de mesures émanant d'un pouvoir politique, le résultat d'un ordre social, d'une relation de genre (rôles homme/femme), ou même de l'interprétation d'une norme grammaticale. Bien que la prise en compte du contexte politique, économique et social qui génère les pratiques répressives et censoriales soit incontournable, les différentes contributions à cet ouvrage ne prennent pas tant pour objet ces pratiques elles-mêmes que les effets qu'elles produisent, que ce soit sur les agents de la vie politique et culturelle, sur les œuvres et leur économie 
interne, ou sur leur diffusion. Si la censure est subie, elle est surtout considérée comme génératrice d'effets : « la censure est une manière de produire des discours, d'imposer à l'avance des contraintes quant à ce qui sera ou ne sera pas un discours acceptable " écrit Judith Butler (Le pouvoir des mots. Discours de haine et politique du performatif, 2008). Elle est considérée comme un phénomène universel et polymorphe, partie intégrante des sociétés humaines qui, loin de se limiter au champ politique à propos duquel elle est le plus souvent étudiée, reste d'une incontournable actualité si l'on tient compte des limitations de la liberté d'expression dues à la répartition sociale des pouvoirs et des rôles et aux effets de la censure psychique théorisée par la psychanalyse qui fournit des outils pour l'étude des formes de langages obliques ("Censure et mémoire selon Freud», in «Censure et mémoire. «La noche de los visones » de Pedro Lemebel », de Mónica Zapata).

La réflexion progresse de l'étude des pratiques politiques à celle des pratiques textuelles. Une première partie intitulée " Pouvoirs et censures de l'Ancien Régime au $\mathrm{XX}^{\mathrm{e}}$ siècle » réunit quatre articles qui envisagent la censure dans des rapports de force institutionnels visant la conquête du pouvoir politique («Affirmation du pouvoir royal et censure dans l'Aragon moderne ", Fausto Garasa), dans le maintien de l'ordre public (« Las limitaciones de la libertad de expresión del anarquismo español a finales del siglo XIX y principios del XX», Antonio Robles Egea) et de l'ordre moral ("Censure et stratégies de contournement. Le cas des publications érotiques clandestines en Espagne ([dernier quart du XIXe-début du XXe siècle ]», Jean-Louis Guereña), ce dernier cas faisant également intervenir le poids du marché. À cela s'ajoute un quatrième article ( L'historien et la censure : La Historia de la Educación en Guatemala de Carlos González Orellana et le régime d'Estrada Cabrera (1898-1920) ", Émilie Mendonça) qui examine les facteurs idéologiques qui génèrent une forme de refoulement dans le récit historiographique libéral guatémaltèque.

La deuxième partie intitulée "Les dictatures face aux productions littéraires et cinématographiques : franquisme, salazarisme, dictatures latino-américaines ", réunit six articles consacrés à la censure culturelle (revues, roman, cinéma, théâtre) en contexte dictatorial. L'originalité de ces sondages est d'explorer ses failles et ses paradoxes. Un moindre contrôle des genres à diffusion réduite, telle la poésie, des protecteurs bien en cour, la mise en avant de valeurs humanistes permirent ainsi à un groupe de poètes cordouans homosexuels de promouvoir en traduction la poésie étrangère et des poètes sentant le soufre dans l'Espagne des années 40 et 50 ( La revista cordobesa Cántico y la censura literaria en la España franquista ", José Reyes de la Rosa). Dans le domaine cinématographique, la censure fut tiraillée entre l'impact des œuvres sur un public national et la répercussion sur l'image de l'Espagne des prix obtenus hors des frontières par des films espagnols. La rigueur entraînée par des considérations internes est illustrée par le sort réservé à Los jueves, milagro («La censura en el cine español del franquismo. El caso de Los jueves, milagro (1957) de Berlanga ", Kepa Sojo Gil) tandis que l'article consacré à la stratégie "possibiliste " («Aux limites du dicible et du visible. Notes sur le cinéma, le "possibilisme” et la culture de l'opposition sous le franquisme ", Jorge Nieto Ferrando) fait apparaitre la marge supplémentaire de liberté d'expression due au poids de l'opinion internationale à la fin des années 60. Le paradoxe surgit encore, au théâtre, de la comparaison entre différentes situations nationales dans des contextes répressifs similaires. Alors que dans le Portugal de Salazar, le théâtre critique est orphelin de public car il ne peut être représenté («Issues de secours. Quand le théâtre portugais s'expatrie pour être vu du 
public. L'État nouveau de Salazar (1933-1974)», Graça Dos Santos), l'Argentine et l'Uruguay ont vu au contraire se développer, dans les failles d'un contexte dictatorial, un théâtre militant, reposant sur la complicité du public, qui n'a vécu que le temps de la dictature ( Censura y movimientos teatrales de resistencia. La dimensión política del arte en tiempos de dictadura (Uruguay y Argentina) ", Lucía Masci). Paradoxale aussi, et ambiguë, est la figure d'Ana Mariscal, actrice vedette du film Raza (1941) dont le scénario fut écrit par Franco, également auteure d'un roman intitulé Hombres (1943), interdit car non conforme à la morale et aux bonnes mœurs (il mettait en scène la liberté sexuelle, le divorce, le suicide). Ana Mariscal se forge rétrospectivement une figure de victime de la censure tout en défendant encore l'institution en 1970: « La censura me parece una higiene social » (« Les paradoxes de la censure, entre le système et l'intime. Hombres (1943), le roman censuré d'Ana Mariscal », Laurence Karoubi).

Il n'en reste pas moins que l'efficacité des institutions censoriales peut être redoutable, comme lorsque l'Inquisition, à l'origine gardienne de l'orthodoxie religieuse, déploya son action au service du pouvoir politique dans l'Espagne moderne (F. Garasa) ou lorsqu'un censeur franquiste, au lieu de supprimer, selon la pratique habituelle, des passages du film de Berlanga Los jueves, milagro, s'autorisa à écrire et à faire tourner par un réalisateur lié à l'Opus Dei une seconde partie dénaturant le film (K. Sojo Gil). On aurait aimé savoir pour quelle raison Berlanga n'a pas purement et simplement retiré son film.

6 La troisième partie, moins homogène, intitulée «Langue et littérature : autocensures, oublis, manipulations ", élargit la question de la censure à d'autres relations de pouvoir. Le régime de parole en contexte répressif est encore présent dans l'article que Stephen G. H. Roberts consacre aux articles politiques - certains d'entre eux en français - publiés en exil par Miguel de Unamuno pendant la dictature de Primo de Rivera. La logique économique l'emporte en revanche sur les risques politiques dans la Cuba actuelle d'après l'article de Paula Martinez « Censure politique ou économique ? Le cas de Leonardo Padura Fuentes, écrivain cubain publiant en Espagne». Les pages consacrées à Semprún («Censures et déplacements dans l'œuvre de Jorge Semprún. Le Grand Voyage et Autobiographie de Federico Sánchez, Émilie Rikir) s'intrègrent moins bien à l'ensemble. Appliquée à la première œuvre, écrite en français comme tous les romans de Semprún sur l'expérience des camps, la notion de censure n'est guère pertinente. Et la seconde, Autobiografía de Federico Sánchez (1977), qui retrace la vie du militant communiste antifranquiste exilé et son expulsion du comité central du PCE en 1964, fait l'objet de considérations psychologiques assez éloignées du sujet.

7 En régime démocratique, la question de la censure se déplace vers un volet idéologique et collectif qui se manifeste par le refoulement de pans de l'histoire nationale. En particulier celle des maquis dans les luttes antifranquistes, qui font retour dans une série de romans publiés en Espagne entre 1984 et 2005, analysés par Christophe Dubois ("L'oubli comme manifestation de la censure dans les "romans de la mémoire" »). La problématique de l'oubli et de la mémoire est reprise par Mónica Zapata («Censure et mémoire. "La noche de los visones" de Pedro Lemebel ») à propos d'une nouvelle publiée en 2000 par l'écrivain chilien et située au moment de la chute du gouvernement de Salvador Allende. La notion de censure est également appliquée à la dialectique du silence et de la parole dans la répartition sociale de celle-ci, signe de relations de pouvoir entre hommes et femmes («Parole, silence et subversion dans La casa verde de Mario Vargas Llosa ", Félix Terrones). Elle est encore abordée dans le champ de la 
norme grammaticale qui énonce ce qui peut se dire et ne doit pas se dire, à propos de phénomènes d'hypercorrection dans les grammaires à l'usage des Français par rapport aux normes édictées par les grammaires espagnoles (« Norme et censure de la norme dans les grammaires de l'espagnol ", Amélie Piel).

8 La notion de déplacement est déclinée dans son volet géographique : exil découlant de la répression politique (A. Robles Egea, S. G.H. Roberts), choix d'une autre langue d'écriture (S. G.H. Roberts), quête d'un public étranger à défaut d'un public national (G. Dos Santos) ou détour par une notoriété internationale pour prévenir une censure locale (J. Nieto Ferrando, P. Martinez). Le recours à des circuits de distribution clandestins et au colportage, comme dans le cas des publications érotiques et pornographiques au début du $\mathrm{XX}^{\mathrm{e}}$ siècle, en est une autre forme (J. L. Guereña). Recourir à la caution de protecteurs, afficher une identité catholique conforme à l'idéologie dominante et dissoudre dans un discours humaniste le contenu subversif des œuvres dont on veut faire la promotion (J. Reyes de la Rosa), c'est opérer de nouvelles formes de déplacement du contenu de l'œuvre vers les circonstances de sa production et de sa diffusion. Le fait de " projeter un contexte conceptuel à la périphérie ou à l'extérieur» (Neuschäffer: Adiós a la España eterna, 1994) devient figure du discours lorsque l'interdit fait retour de manière latérale ou transposée dans l'allusion, la métonymie, la métaphore, le symbole, l'allégorie. Dans le récit littéraire (M.Zapata), cinématographique (J. Nieto Ferrando) et le texte théâtral (L. Masci), comme dans le rêve et autres productions psychiques, le déplacement s'accompagne souvent de condensations grâce auxquelles le récit manifeste est plus laconique que le contenu latent, plusieurs chaînes associatives pouvant interférer en une représentation unique. Ces constantes du psychisme et de la création revêtent une importance particulière en contexte répressif en raison de leurs effets pragmatiques. Le décalage entre sens manifeste et sens latent nécessite de la part du destinataire un travail d'interprétation qui ne peut surgir que sur « un fond de croyances, d'opinions, de sous-entendus » et à condition «de partager le même bagage culturel dissident pour suppléer aux zones d'indétermination des textes » (J. Nieto Ferrando, 156). Le risque encouru est commun à tous les langages obliques, manquer leur cible. En revanche, il a pour effet de conforter et de souder une communauté dissidente (J. Nieto Ferrando, L. Masci) par un langage qui fonctionne comme figure de communion.

Les effets littéraires de la censure ne s'épuisent pas avec la disparition institutionnelle de celle-ci. Le traumatisme de la guerre civile et de la dictature a généré, dans l'Espagne de la Transition, une forme collective de refoulement parfois appelée « amnesia de la Transición » (288). Depuis les années 90, nombre de romanciers espagnols ont mis leur écriture au service du retour du refoulé et œuvrent à reconstruire une mémoire collective (Ch. Dubois). Leurs récits mêlent aux métaphores de l'oubli les processus grâce auxquels le refoulé peut franchir la barrière d'un inconscient collectif, en particulier la fragmentation du récit reconstituant l'anecdote à partir de fragments épars, la polyphonie qui assemble des voix partiellement concordantes, les figures de répétition qui contribuent à fixer les souvenirs. Des procédés, précisons-le, déjà au centre de la poétique de Juan Marsé dans son roman précurseur du «roman de la mémoire", Si te dicen que caí (1975). L'étude de "Noche de los visones» de Pedro Lemebel, qui clôt le volume (M. Zapata), identifie dans la poétique de la nouvelle les mécanismes de condensation, déplacement et symbolisation mis au service d'une chronique des années 80 au Chili qui fait de la maladie - le sida et ses ravages au sein de la communauté homosexuelle au cours de cette décennie - une allégorie de la fin des 
utopies sous les coups de la répression politique et d'une hétérosexualité triomphante. Une esthétique grotesque perçue comme angoissante, ébranlant nos goûts, nos valeurs et l'ordonnancement de notre réalité (Kayser, Lo grotesco. Su configuración en pintura y literatura, 1964), déplace, en conclusion, la question de la censure et des stratégies de contournement du côté de la réception. Ce déplacement de la question de l'Histoire et de la création vers l'impact des œuvres sur le public, qui était déjà présent dans les pages consacrées au cinéma et au théâtre, ouvre de nouvelles perspectives de recherche.

\section{AUTEUR}

\section{GENEVIÈVE CHAMPEAU}

Université Bordeaux Montaigne 